\title{
Multifractal Analysis and Simulation of Rainfall Fields in Space
}

\author{
R. Deidda \\ CRS4, Centro di Ricerca, Sviluppo e Studi Superiori in Sardegna. Via Nazario Sauro 10, \\ I-09123 Cagliari, Italy.
}

\author{
Camera-ready Copy for \\ Physics and Chemistry of the Earth \\ Manuscript-No. B1 \\ Offset requests to: \\ Roberto Deidda \\ CRS4 \\ Via Nazario Sauro, 10 \\ I-09123 Cagliari, Italy.
}




\title{
Multifractal Analysis and Simulation of Rainfall Fields in Space
}

\author{
R. Deidda \\ CRS4, Centro di Ricerca, Sviluppo e Studi Superiori in Sardegna. Via Nazario Sauro 10, I-09123 Cagliari, Italy.
}

Received 15 January 1998 - Accepted 1 May 1998

\begin{abstract}
Statistical downscaling of precipitation from the large scales of meteorological models to the characteristic response scales of small catchment basins needs to correctly preserve the anomalous scaling laws observed in real rainfall. Multifractal behaviour of precipitation in space is investigated on a set of rainfall fields obtained by a high resolution simulation with a limited area model for numerical weather prediction and on two sets of radar measures of rainfall during the GATE campaign. Some sets of synthetic rainfall fields were generated applying a multifractal model based on a wavelet expansion with coefficients extracted by a log-Poisson random cascade, and results of comparisons with the GATE rainfall fields are presented.
\end{abstract}

\section{Introduction}

The recent profusion of centers for numerical weather prediction throughout the world makes available rainfall field predictions that can also be employed to forecast flood events. The prediction of runoff using rainfall fields obtained by meteorological models can be schematically represented with three main steps. The first one is governed by the atmospheric dynamics and is characterized by the evolution of cyclogenetic disturbances and by the formation of large scale precipitation areas. The spatial resolution of precipitation fields distributed by meteorological centers ranges from order of $10^{4} \mathrm{~km}^{2}$ area grid-size for general circulation models (GCMs) to order $10^{2} \mathrm{~km}^{2}$ for limited area models (LAMs). In the second step the large scale precipitation fields obtained as output of meteorological models must be "downscaled" to the the basins' response scales, preserving correctly the statistical properties observed on real signals across the different scales. In the last step the downscaled rainfall is the input of soil-vegetation processes and must be partitioned into runoff, infiltration and evapotranspiration.

Correspondence to: Roberto Deidda
This paper deals with stochastic downscaling of rainfall from the resolution of meteorological models to basins' response scales. In the past essentially two kinds of approaches have been addressed to statistical modeling of precipitation fields within the meso-scale areas: clusterbased models and fractal/multifractal models. Rainfall processes in cluster-based models are commonly organized in a preferred hierarchy of scales in space and time. This hierarchy of scales describes the process of rainband arrival, the cluster organization of cells within a rainband, and the life-cycle of cells belonging to each cluster. A hierarchical description was first designed by LeCam (1961), while a large diffusion of cluster-based models was reached after Waymire, Gupta and Rodriguez-Iturbe proposed the well-known WGR model (Waymire et al., 1984). A disadvantage of these kinds of models is certainly the large number of parameters that need to be tuned, e.g., the WGR model requires up to 10 parameters to describe the hierarchies in space and time in storm development.

More recent research follows the second approach and deals with multifractal models based on random cascades (Gupta and Waymire, 1993; Hubert et al., 1993; Ladoy et al., 1993; Lovejoy and Schertzer, 1992; Over and Gupta, 1994; Schertzer and Lovejoy, 1987; Tissier et al., 1993). Multifractal theory can be considered the most powerful approach to nonlinear and intermittent processes like precipitation itself: if some kind of similarity holds over a range of scales, it allows one to exert a robust statistical control on the moments of a given distribution of measures. Multifractal models can easily capture any moment of the observed signal, especially higher order moments, and so are particularly suitable to correctly reproduce the strongest events. A model recently developed for the construction of positive definite multifractal measures in spaces of any dimension (Deidda, 1997; Deidda et al., 1998) is briefly reviewed here for generation of spatial fields of rainfall. The model is based on an expansion of wavelets with coefficients belonging to a stochastic cascade. The log-Poisson distribution was chosen as random 
MS No.: B1

First author: R. Deidda

generator of the multiplicative process. In such a way the statistical properties of the model can be tuned with only two parameters.

An analysis of statistical properties of rainfall in space is presented: it is shown that precipitation is a strongly intermittent and nonlinear process and that rainfall fields are characterized by anomalous scaling laws. The multifractal analysis was performed on two sets of rainfall spatial fields, based on the radar observations during the GATE campaign (GARP, Global Atmospheric Research Program, Atlantic Tropical Experiment), and on a set of precipitation fields obtained by a numerical simulation at very high resolution $(10 \mathrm{~km})$ performed with a LAM. Multifractal behaviour of GATE rainfall fields was investigated for different durations: original resolution was 15 minutes, but also fields accumulated over 30 minutes, 1 hour, 3, 6, 12 and 24 hours were analyzed. It is shown that the anomalous scaling laws of rainfall in space depend strongly on the time scale over which precipitation is accumulated: rainfall is characterized by a stronger intermittency for shorter accumulation times. The set of LAM precipitation fields, stored every 6 hours of simulation, has shown anomalous scaling laws very close to those found for the GATE sets at the corresponding 6-hour accumulation time.

The final part of this work is dedicated to random generation of rainfall fields with the multifractal model. A number of synthetic fields were generated by downscaling the large scale precipitation of the GATE over squares of $64 \times 64 \mathrm{~km}$ to squares of $4 \times 4 \mathrm{~km}$. The two parameters of the multifractal model were estimated on the 6-hour rainfall depths of the GATE datasets and that of the LAM simulation. Simulated fields were successfully compared to the observed ones. Comparisons were based on the anomalous scaling laws of synthetic and observed rainfall and also on the distribution of precipitation depths on the smaller scale of $4 \times 4 \mathrm{~km}$.

The work in this paper is organized as follows: in section 2 the multifractal model for random generation of synthetic rainfall is briefly reviewed; in section 3 a multifractal analysis of rainfall fields is performed on the two sets of the GATE campaign and on a set of precipitation fields produced by a limited area model simulation; in section 4 the application of the multifractal model to downscale rainfall fields is discussed and results of some synthetic generations are presented; in section 5, the conclusions of this work are drawn.

\section{The model}

The construction of a positive definite measure $\phi(x, y)$ with $(x, y) \in[0,1]^{2}$ is presented. The multifractal field $\phi(x, y)$ is obtained as a wavelet expansion with coefficients extracted by a stochastic cascade:

$$
\phi(x, y)=\sum_{j=0}^{N} \sum_{k_{x}=0}^{2^{j}-1} \sum_{k_{y}=0}^{2^{j}-1} \alpha_{j, k_{x}, k_{y}} \psi_{j, k_{x}, k_{y}}(x, y)
$$

where $j$ is the cascade level index, varying from 0 (first element) to $N$ (number of cascade levels); $k_{x}$ and $k_{y}$ are position indexes on the two axis where the signal is generated; $\psi_{j, k_{x}, k_{y}}(x, y)$ is a wavelet on level $j$ with position indexes $k_{x}, k_{y} ; \alpha_{j, k_{x}, k_{y}}$ is the coefficient extracted from the stochastic cascade.

The two-dimensional wavelets $\psi(x, y)$ are defined as a product of two one-dimensional basis wavelets $\Psi(z)$, positive definite and integrable for $z \in[0,1]$ and zero elsewhere:

$\psi_{j, k_{x}, k_{y}}(x, y)=2^{2 j} \Psi\left(2^{j} x-k_{x}\right) \Psi\left(2^{j} y-k_{y}\right)$

The normalization in modulus of the basis function $\Psi(z)$ assures the normalization of each wavelet $\psi(x, y)$, defined by the above equation. The following Gaussian distribution is an example of a basis wavelet:

$\Psi(z)= \begin{cases}c \exp \left[-\frac{1}{2}\left(\frac{z-\mu}{\sigma}\right)^{2}\right] & z \in[0,1] \\ 0 & z \notin[0,1]\end{cases}$

where $\mu=\frac{1}{2}, \sigma=0.15 \div 0.2$ and $c \cong \frac{1}{\sigma \sqrt{2 \pi}}$ is a normalization constant.

The random cascade is constructed using a multiplicative process; each son $\alpha_{j, k_{x}, k_{y}}$ at the $j$-th level is obtained multiplying the corresponding father at level $j-1$ by an independent and identical distributed random variable $\eta$, called generator:

$\alpha_{j, k_{x}, k_{y}}=\alpha_{j-1, \frac{k_{x}}{2}, \frac{k_{y}}{2}} \eta$

Ensemble averages of $q$-moments of random variables $\alpha$ can be related to the statistics of the generator:

$\overline{\alpha_{j, k_{x}, k_{y}}^{q}}=\overline{\alpha_{j}^{q}}=\alpha_{0}^{q}{\overline{\eta^{q}}}^{j}$

Let $I=\int_{0}^{1} d x \int_{0}^{1} d y \phi(x, y)$ be the integral of the synthetic signal, the first term of the random process must be:

$\alpha_{0}=\frac{I}{\sum_{j=0}^{N} 2^{2 j} \bar{\eta}^{j}}$

Structure functions $S_{q}(r)$ of signals (1) depend only on the ensemble averages of the moments of the generator $\eta$ :

$S_{q}(r)=<\left[\int_{\xi}^{\xi+r} d x \int_{\theta}^{\theta+r} d y \phi(x, y)\right]^{q}>\sim r^{\zeta(q)}$

where the spatial average $\langle\cdots\rangle$ is computed over all starting points $(\xi, \theta)$.

After some computations we can derive the theoretical expectation for the exponents $\zeta(q)$ :

$\zeta(q)=q\left(2+\log _{2} \bar{\eta}\right)-\log _{2} \overline{\eta^{q}}$

The choice of the probability distribution of the random generator $\eta$ characterize the multifractal behaviour of 
Journal: Physics and Chemistry of the Earth

MS No.: B1

First author: R. Deidda

synthetic signals. In this work the log-Poisson distribution was used:

$\eta=e^{H} \beta^{y} \quad ; \quad P(y=m)=\frac{c^{m} e^{-\varepsilon}}{m !}$

where $H$ and $\beta$ are constant parameters, while $y$ is a Poisson distributed random variable with parameter $c: E[y]=c$. The moment of order $q$ of the Log-Poisson distribution is equal to:

$\overline{\eta^{q}}=\exp \left[q H+c\left(\beta^{q}-1\right)\right]$

The expected scaling of signals can be finally evaluated:

$\zeta(q)=2 q+c \frac{q(\beta-1)-\left(\beta^{q}-1\right)}{\ln 2}$

where the multifractal exponent $\zeta(q)$ depend only by parameters $c$ and $\beta$.

\section{Multifractal analysis of rainfall fields}

Statistical properties of rainfall fields were investigated on two sets of radar measures obtained during the GATE campaign and a set of precipitation fields obtained as output of a high resolution numerical simulation, performed with a meteorological LAM (Buzzi et al., 1994), over a memory distributed parallel machine (Marrocu et al., in press). The GATE spatial fields of 15-minute precipitation depths were collected in the eastern Atlantic coast of Africa during two different periods in 1974: the set belonging to the first period from 28 June to 15 July is designated as GATE1 (1715 fields), while the set belonging to the second period from 28 July to 15 August is referred to as GATE2 (1512 fields). Original resolution of fields was $4 \mathrm{~km}$ over a regular square lattice $100 \times 100$, but only a $64 \times 64$ grid centered in each image was used in this work, since data are really provided only within a $200 \mathrm{~km}$ radius of the center of each image. The set of LAM rainfall consists of 15 fields of 6 -hour precipitation depths over a regular grid $256 \times 256$ with a spatial resolution of about $10 \mathrm{~km}$ covering Europe, starting on 0GMT of 19 February 1991 and ending on 18GMT of the

Table 1. Number $N$ of selected fields from GATE1 and GATE2 datasets for the spatial multifractal analysis and estimates of the log-Poisson parameters $\beta$ and $c$ for generation of synthetic spatial fields of rainfall depth at different durations.

\begin{tabular}{||c||c||c|c||c||c|c||}
\hline \multicolumn{1}{||c||}{} & \multicolumn{3}{c||}{ GATE1 } & \multicolumn{3}{c||}{ GATE2 } \\
\hline duration & $N$ & $\beta$ & $c$ & $N$ & $\beta$ & $c$ \\
\hline 15 min & 436 & 0.42 & 1.00 & 316 & 0.38 & 1.00 \\
\hline 30 min & 215 & 0.43 & 0.92 & 154 & 0.40 & 0.92 \\
\hline 1 hour & 128 & 0.44 & 0.83 & 95 & 0.41 & 0.88 \\
\hline 3 hours & 70 & 0.44 & 0.73 & 52 & 0.44 & 0.81 \\
\hline 6 hours & $\mathbf{4 6}$ & $\mathbf{0 . 4 8}$ & $\mathbf{0 . 7 7}$ & $\mathbf{4 0}$ & $\mathbf{0 . 4 6}$ & $\mathbf{0 . 7 5}$ \\
\hline 12 hours & 25 & 0.55 & 0.78 & 26 & 0.51 & 0.77 \\
\hline 24 hours & 13 & 0.62 & 0.71 & 14 & 0.56 & 0.68 \\
\hline
\end{tabular}

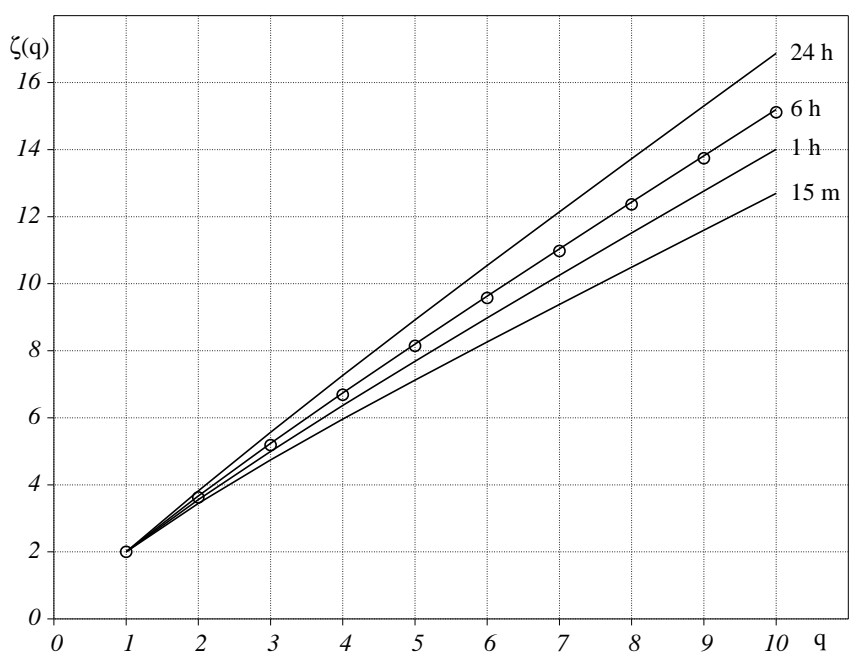

Fig. 1. Averages of exponents $\zeta(q)$ of GATE1 and GATE2 datasets for 15-minute, 1-hour, 6-hour, and 24-hour durations (solid lines) are compared with the averages of the multifractal exponents estimated from LAM 6-hour precipitation fields (circles).

22 of the same month. Structure functions were computed for each rainfall field $\phi(x, y)$ using equation (7).

A multifractal analysis was performed on the GATE rainfall fields at the original time resolution of 15 minutes and also on the cumulated rainfall fields over 30 minutes, 1 hour, 3, 6, 12, and 24 hours. Two criteria were applied to select the fields with a significant rainy area and to assure scaling properties with a minimum statistical significance on exponents $\zeta(q)$ : the first criterion requires that at least $10 \%$ of grid points have a nonzero measure of precipitation, the second criterion requires a minimum correlation coefficient, fixed at the level 0.995, for the linear regression between the logarithms of structure functions $S_{10}(r)$, defined by equation (7), and the logarithms of spatial scales $r$ from 4 to 64 $\mathrm{km}$, regularly sampled in the logarithmic scale with integer powers of 2 .

The number $N$ of fields selected for each duration considered in the analysis from GATE1 and GATE2 sets with the two criteria discussed above can be found on Table 1 . The first 10 multifractal exponents $\zeta(q)$ were then estimated on selected fields in the range of scales from 4 to $64 \mathrm{~km}$. In Figure 1 are compared the sample averages of exponents $\zeta(q)$ at different durations from GATE1 and GATE2 sets. In Table 1 are summarized the corresponding values of the model parameters $\beta$ and $c$ obtained by imposing sample exponents $\zeta(3)$ and $\zeta(8)$ in equation (11). A more detailed discussion on results of the multifractal analysis of GATE rainfall fields can be found in Deidda et al. (1998).

The multifractal analysis of LAM 6-hour rainfall was applied only on fields satisfying the criterion of minimum correlation coefficient $(>0.995)$ for the linear regression between the logarithms of structure functions $S_{10}(r)$ and the logarithms of spatial scales $r$ ranging from 10 to $160 \mathrm{~km}$, sampled with integer powers of 2 . With this criterion 13 of the 15 available precipitation fields were selected: in Figure 
MS No.: B1

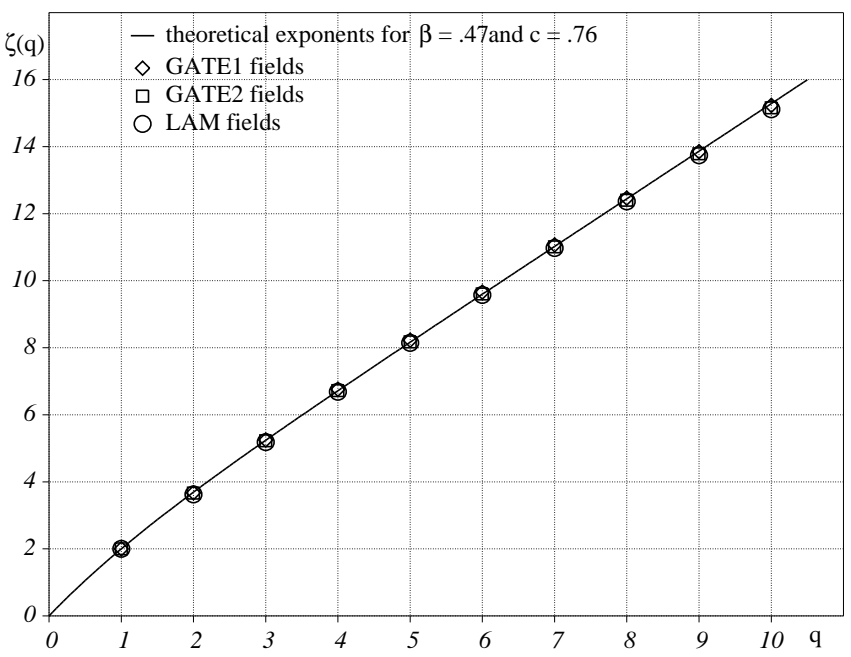

Fig. 2. Sample averages of exponents $\zeta(q)$ for 6-hour precipitation depths estimated on GATE1, GATE2 and LAM fields are compared with the theoretical scaling (11) of the multifractal model with log-Poisson parameters $\beta=0.47$ and $c=0.76$.

1 sample averages of the first 10 exponents $\zeta(q)$ are drawn with circles for comparison with the corresponding scaling of 6-hour GATE rainfall.

Multifractal exponents $\zeta(q)$ from GATE1, GATE2, LAM sets of 6-hour rainfall depths are also plotted in Figure 2 and compared with the theoretical scaling (11) of the model described in previous section with parameters $\beta=0.47$ and $c=76$ : this couple of values will be used in the next section to generate some sets of synthetic rainfall fields.

This analysis gives evidence of the dependence of the spatial statistical properties of the precipitation fields on time duration, in which rainfall is accumulated: multifractality of precipitation fields is more pronounced for short accumulation times. Another important result is the substantial coherence on the exponents $\zeta(q)$ determined for the 6-hour accumulated precipitation fields based on LAM simulations and that estimated by GATE dataset at the same duration. Although the parameterization of rainfall processes in meteorological models are heavily simplified and despite the fact that the GATE and LAM datasets belong to different seasons and geographical regions, the numerical simulation with the LAM has produced precipitation fields which statistical properties are in good agreement with that observed in the GATE radar measurements. Results of the multifractal analysis seem to show some kind of "universality" of the statistical properties of rainfall in space.

\section{Simulations of synthetic rainfall fields}

Some random simulations of synthetic rainfall fields generated with the model described on section 2 are analyzed here in view of potential applications of this procedure to downscaling large scale forecasts produced by meteorological centers for numerical weather prediction. Model parameters were set to $\beta=0.47$ and $c=0.76$ :
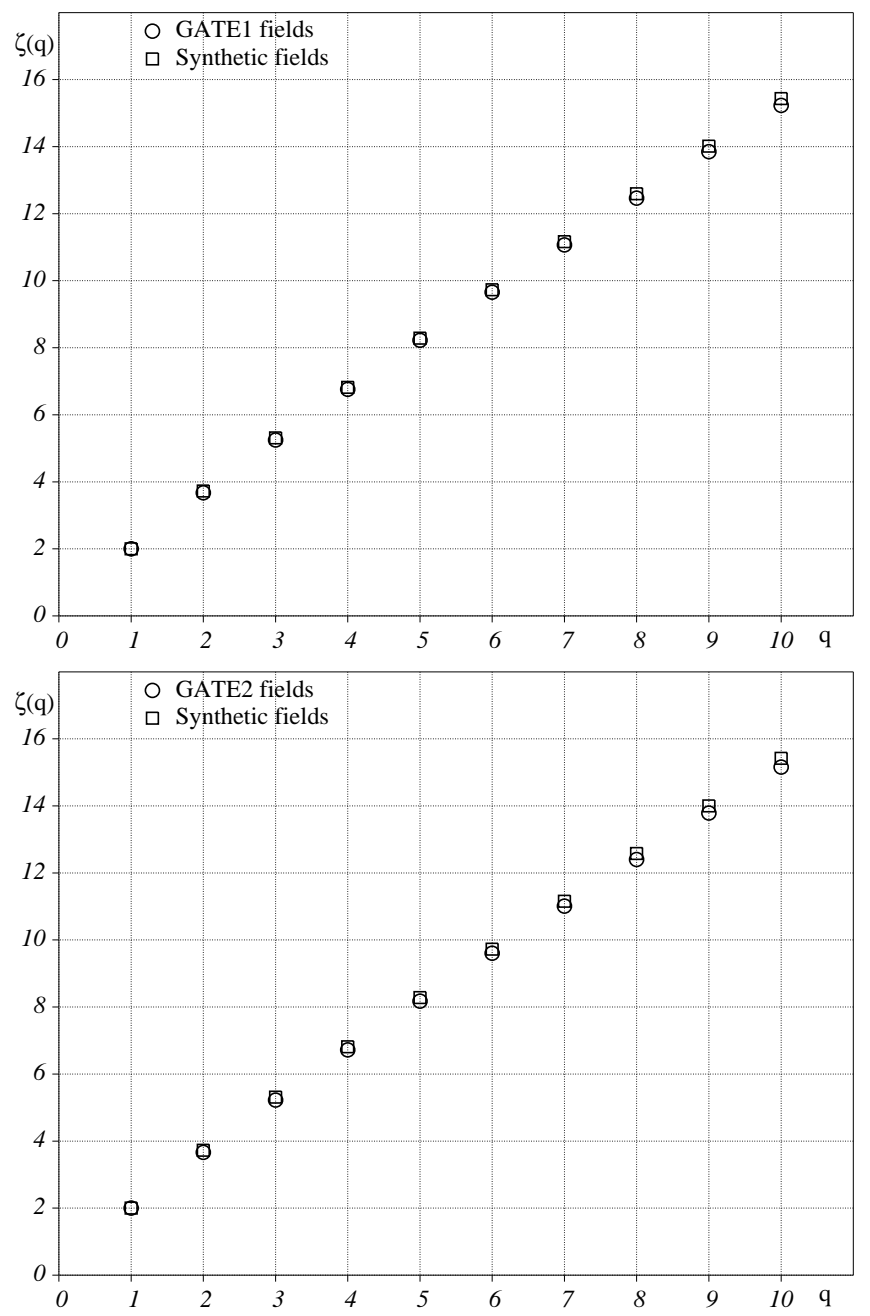

Fig. 3. First 10 mean exponents $\zeta(q)$ for 6-hour precipitation depths estimated on GATE1 (top) and GATE2 (bottom) fields are compared with the exponents estimated on the random rainfall fields.

with these values, the theoretical scaling (11) fit with good accuracy the sample exponents $\zeta(q)$ estimated both on the two sets of GATE 6-hour precipitation fields and on the set of LAM rainfall frames as already noted (see Figure 2).

Before generating synthetic rainfall, each GATE 6-hour precipitation field was divided into a $4 \times 4$ grid of $64 \times 64 \mathrm{~km}$ sub-boxes. The model was then applied to downscale rainfall observed over each $64 \times 64 \mathrm{~km}$ sub-box to smaller boxes of $4 \times 4 \mathrm{~km}$ : final resolution of each random field was the same as GATE. In such a way the downscaling was performed in the same range of scales where the anomalous scaling was observed on GATE fields and where model parameters $\beta$ and $c$ were estimated.

200 sets of synthetic rainfall were randomly generated starting from the large scale precipitation observed on the $64 \times 64 \mathrm{~km}$ sub-boxes of GATE1 and GATE2 datasets. Each random set was composed of the same number of fields selected during the multifractal analysis of the previous section (see Table 1): e.g., each one of the 100 sets based on GATE1 rainfall is constituted by 46 fields. 

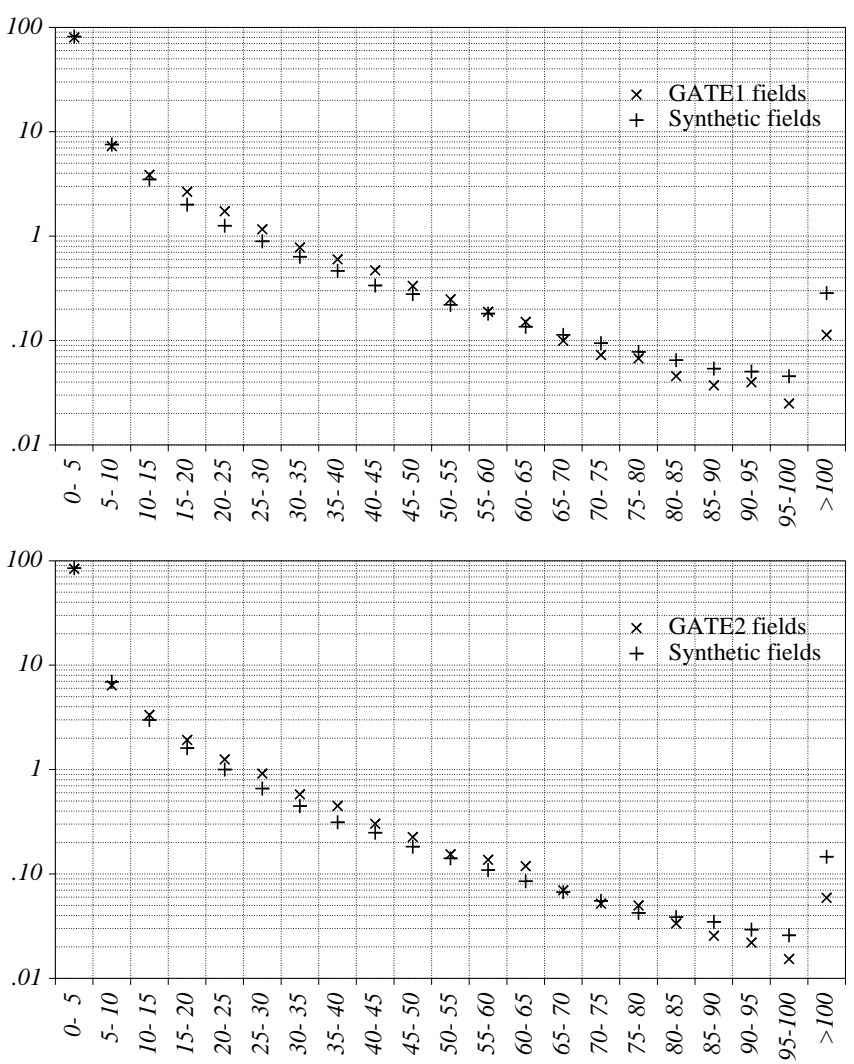

Fig. 4. Rainfall distributions in GATE1 (top) and GATE2 (bottom) fields are compared with synthetic fields: histograms display percentages of elementary $4 \times 4 \mathrm{~km}$ cells with precipitation depths belonging to $5 \mathrm{~mm}$ classes.

Anomalous scaling laws of synthetic rainfall fields were successfully compared with the multifractal behaviour observed in GATE data. Figure 3 shows that statistical properties of generated fields are very similar to that of GATE: sample exponents $\zeta(q)$ are the averages over 46 and 40 fields, respectively for GATE1 and GATE2, while $\zeta(q)$ estimated on synthetic fields were averaged respectively over $46 \times 100$ and $40 \times 100$ fields. Another validation of the downscaling procedure is presented in Figure 4: histograms show the percentages of elementary $4 \times 4 \mathrm{~km}$ cells with precipitation depths belonging to $5 \mathrm{~mm}$ classes. Although there are discrepancies for the rarer events plotted in the right-hand tail that may be due to the small number of samples in the higher classes, the body of distributions of small scale precipitation depths in random fields is very close to that of the real rainfall observed in GATE.

\section{Conclusions}

Flood forecasts can be performed coupling meteorological predictions of rainfall fields to hydrological rainfall-runoff models. In doing so it is necessary to fill the gap between the large scale over which meteorological fields are solved and the natural scales of basins' response: this is referred as the "downscaling" problem. In this paper it was shown how multifractal techniques can be applied to transfer down to smaller scales the information of the large scale precipitation produced by numerical weather prediction models. A multifractal model based on a wavelet expansion with coefficients extracted by a stochastic cascade was reviewed for random generation of spatial rainfall fields. The log-Poisson distribution was chosen as generator of the multiplicative cascade. Anomalous scaling laws of synthetic signals depend only on the two parameters $\beta$ and $c$ of the log-Poisson distribution.

The multifractal analysis on two sets of precipitation fields derived by radar observation during the GATE campaign has shown that statistical behaviour of precipitation in space is dependent on the time duration over which rainfall is accumulated: precipitation is more intermittent for shorter time durations. Results on another set of 6-hour rainfall fields obtained as output of a LAM integration confirm the anomalous scaling found on GATE data at the same duration: this is not a trivial result considering the gross parameterizations of microphysical processes that are commonly assumed in meteorological models.

For synthetic generation of spatial precipitation fields, parameters $\beta$ and $c$ of the multifractal model were estimated on the GATE and LAM sets of 6-hour rainfall. The model was then successfully employed to downscale GATE precipitation fields starting from boxes of $64 \times 64 \mathrm{~km}$ to boxes of $4 \times 4 \mathrm{~km}$, corresponding to the smaller resolution of GATE scans. In such a way 200 sets of synthetic rainfall were generated by applying the downscaling procedure in the range of scales where GATE fields have displayed scaling properties: each random set was composed by the same number of fields belonging to GATE1 or GATE2 datasets and used for the multifractal analysis. In addition, it must be underlined that currently ECMWF (European Centre for Medium-Range Weather Forecasts) provides forecasts of precipitation every 6 hours with a spatial resolution of about $60 \mathrm{~km}$ at mid-latitude: this spatial scale is very close to that used here to start the downscaling process.

The multifractal analysis on the 200 sets of synthetic rainfall have displayed the same anomalous scaling laws as observed in the GATE fields. The comparisons between generated and observed distributions of rainfall depths over boxes of $4 \times 4 \mathrm{~km}$ was also successful. Results in downscaling 6-hour rainfall fields have shown that the multifractal model preserves the multifractal behaviour and the statistical properties of the observed rainfall at the same duration. The application of the model to downscale rainfall fields at different duration must be performed with the value of $\beta$ and $c$ parameters estimated on real rainfall at the same duration.

Acknowledgements. This research was supported by the Sardinia Regional Authorities.

\section{References}

Buzzi, A., Fantini, M., Malguzzi, P., and Nerozzi, F., Validation of a limited area model in case of mediterranean cyclogenesis: surface fields and 
MS No.: B1

First author: R. Deidda

precipitation scores., Meteorol. Atmos. Phys., 53, 137-153, 1994.

Deidda, R., Simulazione numerica delle proprietà statistiche dei campi di precipitazione, Ph.D. thesis, University of Padua (Italy), 1997.

Deidda, R., Benzi, R., and Siccardi, F., Multifractal modeling of anomalous scaling laws in rainfall, submitted to Water Resour. Res., 1998.

Gupta, V. K. and Waymire, E. C., A statistical analysis of mesoscale rainfall as a random cascade., J. Appl. Meteor., 32, 251-267, 1993.

Hubert, P., Y.Tessier, Lovejoy, S., Schertzer, D., Schmitt, F., Ladoy, P., Carbonnel, J. P., Violette, S., and Desurosne, I., Multifractals and extreme rainfall events., Geophys. Res. Lett., 20(10), 931-934, 1993.

Ladoy, P., Schmitt, F., Schertzer, D., and Lovejoy, S., Variabilité multifractale des observations pluvimétriques à Nîmes, C. R. Acad. Sci. Paris, 317, 775-782, 1993.

LeCam, L., A stochastic description of precipitation., in 4th Berkeley Symposium on Mathematical Statistics and Probability. vol. 3., pp. 165-186, University of California, Berkeley, 1961.

Lovejoy, S. and Schertzer, D., Multifractals and rain., in New Uncertainty
Concepts in Hydrology and Water Resouces., edited by Z. W. Kunzewicz, vol. 235, University Press, Cambridge, 1992.

Marrocu, M., Scardovelli, R., and Malguzzi, P., Parallelization and performance of a meteorological Limited Area Model, Parallel Computing, in press.

Over, T. M. and Gupta, V. K., Statistical analysis of mesoscale rainfall: dependence of a random cascade generator on large-scale forcing., $J$. Appl. Meteor., 33, 1526-1542, 1994.

Schertzer, D. and Lovejoy, S., Physical modeling and analysis of rain and clouds by anysotropic scaling of multiplicative processes, J. Geophys. Res. D., 92,9693-9714, 1987.

Tissier, Y., Lovejoy, S., and Schertzer, D., Universal multifractals: theory and observations for rain and clouds, J. Appl. Meteor., 32(2), 223-250, 1993.

Waymire, E., Gupta, V. K., and Rodriguez-Iturbe, I., A spectral theory for rainfall intensity at the meso- $\beta$ scale., Water Resour. Res., 20(10), $1453-1465,1984$. 